\title{
XXXIX. Notes on the practical application of the theory of magnetic disturbance by earth-currents
}

\section{R. T. Glazebrook D.Sc. F.R.S.}

To cite this article: R. T. Glazebrook D.Sc. F.R.S. (1901) XXXIX. Notes on the practical application of the theory of magnetic disturbance by earth-currents, Philosophical Magazine Series 6, 1:4, 432-442, DOI: $10.1080 / 14786440109462630$

To link to this article: http://dx.doi.org/10.1080/14786440109462630

册 Published online: 08 Jun 2010.

Submit your article to this journal $[\pi$

Џ Article views: 2

Q View related articles $\square$ 


\section{[ 432$]$}

XXXIX. Notes on the Practical Application of the Theory of Magnetic Disturlance by Earth-Currents. By R. 'T. GLazeBRooK, D.Se., F.R.S.*

THE following notes contain some applications of Professor Rücker's theory.

In fig. 1 let $\mathrm{MBA}$ be the line, $O$ the observatory, $B$ the power-house, $\mathrm{P}$ the tram, and OM perpendicular on the line from 0 .

Then $\mathrm{OM}=y, \mathrm{MB}=b, \mathrm{BP}=x, \mathrm{BA}=a$. Let $\mathrm{OB}=r_{1}$, $\mathrm{OA}=r_{2}$.

Fig. 1.

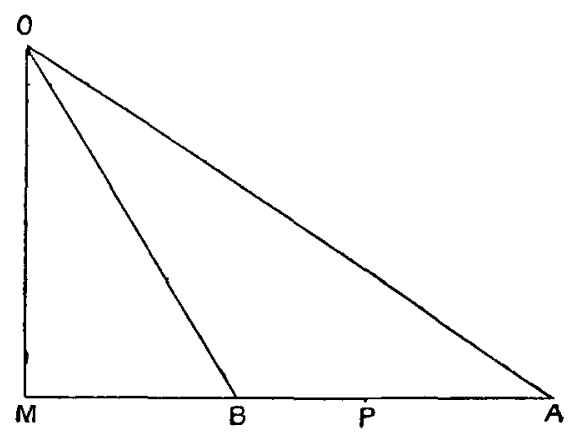

Prof. Rücker's expression for the vertical force at $O$ due to an element $d x$ is

$$
\begin{gathered}
\mathrm{I} y \int_{0}^{a}\left\{1-\frac{\epsilon^{\mu x}+\epsilon^{\mu(a-x)}}{1+\epsilon^{\mu a}}\right\} \frac{d x}{\left\{(b+x)^{2}+y^{2}\right\}^{\frac{3}{2}}}, \\
\mu^{2}=h / k=\text { exterior conductivity/interior conductivity. }
\end{gathered}
$$

Now it will be shown that for many cases as a first approximation $\mu^{2} a^{2} / 4$ mav be neglected compared with unity.

On expanding in powers of $\mu$ and neglecting $\mu^{2} a^{2} / 4$ in small terms, we find

$$
\frac{\mathrm{F}_{v}}{\mathrm{I} y}=\frac{\mu^{2}}{2} \int_{0}^{a} \frac{x(a-x)}{\left\{(b+x)^{2}+y^{2}\right\}^{\frac{3}{2}}} d x ;
$$

and this evaluates to

$$
\begin{aligned}
& \frac{\mu^{2}}{2}\left[\frac{1}{y^{2}}\left\{(a+b)\left(b^{2}+y^{2}\right)^{\frac{1}{2}}-b\left(\overline{a+b^{2}}+y^{2}\right)^{\frac{1}{2}}\right\}-\log \frac{\left.a+b+\sqrt{\left(a+b^{2}+y^{2}\right.}\right)}{b+\bar{b}^{2}+y^{2}}\right], \\
& \text { or } \quad \frac{\mu^{2}}{2}\left[\frac{(a+b) r_{1}-b r_{2}}{y^{2}}-\log \frac{a+b+r_{2}}{b+r_{1}}\right] .
\end{aligned}
$$

* Communicated by the Physical Society : read Dec. 14, 1900. 
The Theory of Magnetic Disturbance by Earth-Curvents. 433

If we take the case in which $b=-\frac{a}{2}, r_{1}=r_{2}=r$, so that the arrangement (fig. 2) is as at Stockton,

$$
\mathrm{F}_{v}=\frac{\mu^{2}}{2} \mathrm{I}\left[\frac{a r}{y}-y \log \left(\frac{r+\frac{a}{2}}{r-\frac{a}{2}}\right)\right] .
$$

Fig. 2.

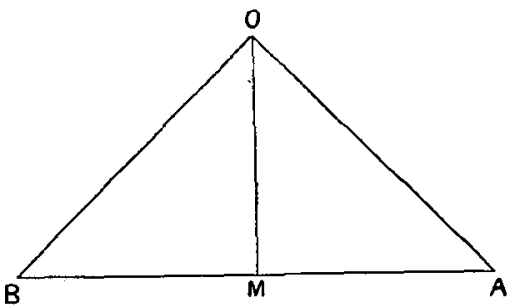

From this equation the vertical force can be calculated. It is, however, somewhat complex for nse. I have, therefore, examined the case in which it is supposed that the difference between flow and return current is constant aloug the line, the whole leak occurring at the sink. In Professor Ruicker's language, I have used the equivalent uniform current.

In this case if $c$ be the leakage coefficient, which is the same as Prof. Rücker's $1-\mathrm{L}$,

$$
\mathrm{F}_{v}=\frac{\mathrm{I} k}{y}\left\{\frac{a+b}{r_{2}}-\frac{b}{r_{1}}\right\}
$$

using the same notation; or taking the above symmetrical case

$$
\mathrm{F}_{v}=\frac{\mathrm{I} \kappa}{y} \frac{a}{r}
$$

In this same case

$$
\mathrm{F}_{h}=\frac{\mathrm{I} \boldsymbol{k} a}{r^{2}} .
$$

Now as to the value of $\kappa$.

We have from the theory developed by Mr. Parry and Prof. Rücker,

$$
x=\frac{\left(1-\epsilon^{-\frac{\mu a}{2}}\right)^{2}}{1+\epsilon^{-\mu a}}
$$

and from the Stockton experiments $\mu \alpha / 2$ is about 64 and $a$ is 2 miles. 


\section{Dr. Glazebrook on the Practical Application of the}

Hence expanding as far as $\{\mu a / 2\}^{4}$, we find

$$
\kappa=\frac{\mu^{2} a^{2}}{8}\left(1-\frac{5}{12} \frac{\mu^{2} a^{2}}{4}+\ldots\right)
$$

or neglecting $\frac{5}{48} \mu^{2} a^{2}$ in comparison with unity,

and

$$
\kappa=\frac{\mu^{2} a^{2}}{8},
$$

$$
\mathrm{F}_{v}=\frac{\mu^{2} \mathrm{I}}{8} \frac{a^{3}}{r y}=\frac{1}{4} \mu^{2} \mathrm{I} \frac{a^{2}}{y} \frac{a}{\sqrt{a^{2}+4 y^{2}}} \ldots .
$$

It is clear that if $a$ is 2 miles, the value of $\kappa$ is about 20 per cent. too great.

To compare these two formulæ I. and II. we may, since a/ $2 r$ is less than unity, expand the logarithm in I.; we thus ohtain

$$
\mathrm{F}_{v}=\frac{\mu^{2} \mathrm{I}}{\mathrm{s}} \frac{a^{3}}{r y}\left\{1-\frac{1}{3} \frac{y^{2}}{r^{2}} \ldots\right\} \text {. }
$$

In the Stockton experiments $a=2, y=\cdot 4, r=2 \cdot 04$; and the term neglected is about 02 .

A result of considerable importance may be deduced from the above formulæ.

It is clear that for similar circuits similarly placed the vertical force varies as the linear dimensions of the circuit multiplied by the current. Now if the number of cars per mile of track is to remain constant, the current most vary as the length of the track. Hence the force is proportional to the square of the linear dimensions.

Fig. 3.

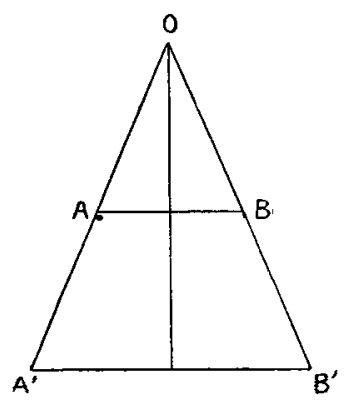

Thus if we have two parallel lines $A B, A^{\prime} B^{\prime}$ (fig. 3), of which $\mathrm{A}^{\prime} \mathrm{B}^{\prime}$ is double the length of $A B$ and at double the distance from the source, the effect of $A^{\prime} B^{\prime}$ is four times that of $\mathrm{AB}$. This is readily seen from first principles. 
Theory of Magnetic Disturbance ly Earth-Currents. 435

For we have clearly

$$
\frac{\mathrm{F}_{n}}{\mathrm{~F}^{\prime}}=\frac{\kappa \mathrm{I}}{y} \times \frac{y^{\prime}}{\kappa^{\prime} \mathbf{I}^{\prime}} .
$$

Also, since the number of cars per mile is constant,

Moreover,

$$
\frac{\mathrm{I}}{y}=\frac{\mathrm{l}^{\prime}}{y^{\prime}} \text {. }
$$

$$
\frac{\kappa}{\kappa^{\prime}}=\frac{y^{2}}{y^{12}} .
$$

Hence

$$
\frac{\mathrm{F}}{\mathrm{F}^{\prime}}=\frac{\kappa}{\kappa^{\prime}}=\frac{y^{2}}{y^{\prime 2}}=\frac{1}{4} .
$$

Various interesting results may be obtained from the simple formula (II.), which we may write

$$
\mathrm{F}_{v}=\frac{1}{4} \mu^{2} a^{2} \mathrm{I} \frac{1}{y} \frac{a}{\sqrt{\left(4 y^{2}+a^{2}\right)}} .
$$

Now if the number of cars per mile be constant the current varies as the length of the line. Hence

and

$$
\mathrm{I}=\lambda a \text {; }
$$

$$
\mathrm{F}_{v}=\frac{1}{4} \lambda \mu^{q} a^{3} \cdot \frac{1}{y} \cdot \frac{a}{\sqrt{4 y^{2}+a^{2}}} .
$$

In this expression the distance $y$ must be measured in centimetres in order to give $F_{v}$ in absolute units. Hence we must introduce in the denominator a factor $1.6 \times 10^{5}$, or in the numerator $62 \times 10^{-5}$. Thus

$$
\mathrm{F}_{0}=\frac{\cdot 62}{4} 10^{-5} \frac{\lambda \mu^{2} a^{3}}{y} \frac{a}{\sqrt{\left(4 y^{2}+a^{2}\right)}},
$$

where the lengths are now all in miles. $10^{-5}$ is an ordinary unit in terrestrial magnetic work denoted by $\gamma$.

With regard to the dimensions of this expression it must be remembered that $\mu^{2} a^{2}$ is a number and $\lambda a$ an electric current. Thus $\mu^{2}$ and $\lambda$ both have dimensions in space, and in the values given for the two quantities it is assumed that each is referred to a mile run of line.

Now according to Professor Rücker's Stockton experiments the approximate value of $\mu a / 2$ is $\cdot 64$, where $\iota=2$ miles.

Hence

$$
\mu=\cdot 64, \quad \mu^{2}=\cdot 41 .
$$




\section{Dr Glazebrook on the Practical Application of the}

We may compare these figures with those given by Mr. Parry in his paper in the 'Electrician' for August 10, 1900. He takes $h$ and $k$ to refer to the exterior and interior conductivity per unit surface and unit volume respectively, and assumes one inch as his unit of length. In his formula $\mathrm{A}$ is the area of the cross section of the rail, and L the area of the surface in contact with the ground per inch run of rails. Thus his quantity $\mathrm{L} h / \mathrm{A} k$ corresponds to my $h^{\prime} k$, with this difference, that his unit of length is the inch, mine the mile; and he finds as the value of $\{\mathrm{L} h / \mathrm{A} k\}^{\frac{1}{2}}$ the number $1 / 180000$. Multiplying this by the number of inches in a mile, we find as the value of $\mu$ the quantity $\cdot 35$, while the leak in a line 1 mile long would be $\cdot 015$. Some other experiments give $\mu=\cdot 425$ and $\mu^{2}=\cdot 18$.

The ratio of the leak to the inflowing current in a line a mile long, which is given by $\mu^{2} / 8$, will in these two cases be $\cdot 051$ and $\cdot 0225$ respectively. By way of illustration 1 have made some calculations on the assumption that the leak for 1 mile is 5 per cent.; so that $\frac{1}{8} \mu^{2}=\cdot 05$ and $\mu^{2}=\cdot 4$, the value found at Stockton. In this case the term neglected in the original expansion is $\frac{1}{24} \times a^{2}$.

Let us also assume that there are 8 cars in a mile each taking rather under 20 amperes, so that

$$
\lambda=150 \text { amperes }=15 \text { c.G.s. units. }
$$

Hence we have

$$
\lambda \mu^{2}=6 .
$$

Then in this case

$$
\frac{\mathrm{F}_{v}}{\gamma}=.93 \times \frac{a^{4}}{y\left\{4 y^{2}+a^{2}\right\}^{\frac{1}{2}}} .
$$

And if we take a line a mile long at a distance of 1 mile from the observatory, we bave

$$
a=1, \quad y=1, \quad \mathrm{~F}_{v}=\cdot 42 \times \gamma .
$$

For the values at Stockton $F_{v}$ is equal to $6 \cdot 2 \gamma$, which is rather less than the $7 \gamma$ actually found.

Instead of working with the current we may use the P.D. at the ends. Let this be V.

Then we can show that

$$
\begin{aligned}
\text { I eakage current } & =\mu k \frac{\mathrm{V}}{2} \frac{1-\epsilon^{-\frac{\mu a}{2}}}{1+\epsilon^{\frac{\mu a}{2}}} \\
& =\frac{h a \mathrm{~V}}{8}
\end{aligned}
$$

to the same approximation. 
Hence

$$
\mathrm{F}_{v}=\frac{1}{4} \frac{h \mathrm{~V}}{y} \frac{a^{2}}{\sqrt{4 y^{2}}+a^{2}} .
$$

Now if the number of cars per mile is given $V$ varies as $a^{2}$; put $\mathrm{V}=v a^{2}, v$ is the potential-difference between two points 1 mile apart.

$$
\begin{aligned}
& \text { Hence total leak in length } a=\frac{h \nu a^{3}}{\gamma} . \\
& \text { But total . . . . . . }=\frac{\lambda \mu^{2} a^{3}}{y} .
\end{aligned}
$$

Therefore $h \nu=\lambda \mu^{2}$, an obvious result when the meaning of the quantities is considered, and

$$
\mathrm{F}_{v}=\frac{h v a^{4}}{4 y \sqrt{4 y^{2}+a^{2}}} \times 62 \times 10^{-5}
$$

if the quantities be all in miles.

A gain we can eliminate $a$ from these equations either by the use of the equation $\mathrm{I}=\lambda a$, or by the use of the equation $\mathrm{V}=\nu a^{2}$.

And we have the following four identical equations :-

$$
\begin{aligned}
\mathrm{F}_{v} & =\frac{\cdot 62}{4} \lambda \mu^{2} \frac{a^{4}}{y} \frac{1}{\sqrt{4 y^{2}+a^{2}}} \times 10^{-5} \\
& =\frac{\cdot 62}{4} \frac{\lambda \mu^{2}}{y}\left(\frac{\mathrm{I}}{\lambda}\right)^{4} \frac{1}{\sqrt{\left(4 y^{2}+\left(\frac{1}{\lambda}\right)^{2}\right)}} \times 10^{-5} \\
& =\frac{62}{4} h v \frac{a^{4}}{y} \frac{1}{\sqrt{\left(4 y^{2}+a^{2}\right)}} \times 10^{-5} \\
& =\frac{.62}{4} \frac{h \nu}{y}\left(\frac{\mathrm{V}}{v}\right)^{2} \frac{1}{\sqrt{\left(4 y^{2}+\frac{V}{v}\right)}} \times 10^{-5} .
\end{aligned}
$$

If we wish to find the connexion between the length of the line and its distance from the observatory, so that when its effect is a maximum it may be less than a given quantity, $\delta$ say, then we must have the right-hand side of the above equations less than $\delta$.

Now we have seen that $\lambda \mu^{2} / 8$ is the maximum leak from 1 mile of the line. Let us call this L. Then

$$
\lambda \mu^{2}=8 \mathrm{~L} \text {; }
$$


and our equation of condition becomes

$$
1 \cdot 24 \times \mathrm{L} \times \frac{a^{4}}{y} \frac{1}{\sqrt{a^{2}+4 y^{2}}} 10^{-5}<\delta .
$$

From this we obtain the rule :-

Calculate in absolute units the leak in a rail 1 mile long when carrying the maximum current required to drive the maximum number of cars in the mile. Let the result be L c.G.s. units of current. Then $a$ must not exceed the valne given by the equation

$$
1 \cdot 24 \times \mathrm{L} \times \frac{a^{4}}{y} \frac{1}{\sqrt{\left(a^{2}+4 y^{2}\right)}} 10^{-5}=\delta ;
$$

or if $\overline{\mathrm{L}}$ be the leak in amperes,

Hence

$$
\overline{\mathrm{L}}=10 \mathrm{~L} \text {. }
$$

$$
\cdot 124 \overline{\mathrm{L}} \times \frac{a^{4}}{y} \frac{1}{\sqrt{\left\{a^{2}+4 y^{2}\right\}}} 10^{-5}=\delta .
$$

If we take $\delta=\frac{1}{2} \gamma$ (a usual case in practice), this reduces to

$$
25 \times \bar{L} \times \frac{a^{4}}{y} \frac{1}{\sqrt{\left(a^{2}+4 y^{2}\right)}}=1 \text {. }
$$

With the numbers already assumed,

and

$$
\lambda \mu^{2}=60 \text { amperes, }
$$

$$
\mathrm{L}=7 \cdot 5 \text { amperes }=5 \text { per cent. of current. }
$$

If we know the resistance of a mile of the track we can calculate the potential-difference required to drive the current through, for it is clear from Mr. Parry's curves and from the theory that for a track 1 mile in length the drop required is given by the product of the current and resistance to a sufficient degree of approximation.

Now for a rail weighing $92 \mathrm{lbs}$. to the mile we may take as the resistance of a mile of continuous rail the value 0.0525 ohm. Thus, allowing for the bonds, the rails being in 36-feet lengths, about one-fortieth of the total resistance, we get as the resistance of a mile of single rail $0.0538 \mathrm{ohm}$. Hence the resistance of a double track will be $0.01 .35 \mathrm{ohm}$, and the potential-difference required to produce a current of 150 amperes will be about 2 volts. This, then, is the value of $\nu$ in the above formula. 
Theory of Magnetic Disturbanee by Earth-Currents. 439)

We thus obtain the following table of values of $a$ and $V$.

Table I.

\begin{tabular}{|c|c|c|}
\hline$y \cdot$ & $a$. & V. \\
\hline 1 & $1 \cdot 05$ & $2 \cdot 25$ \\
2 & $1 \cdot 45$ & $4 \cdot 25$ \\
3 & $1 \cdot 78$ & $6 \cdot 25$ \\
4 & $2 \cdot 05$ & $8 \cdot 5$ \\
5 & $2 \cdot 30$ & $10 \cdot 5$ \\
\hline
\end{tabular}

Or, if wo take $\mu^{2} / 8=0225$, so that the leak in 1 mile is 21 per cent., the current being 150 amperes for each mile of track, and the resistance of a mile of track $0.0135 \mathrm{ohm}$ as before, we have :-

TABLE II.

\begin{tabular}{|c|c|c|}
\hline$y$. & $a$. & $\mathrm{V}$. \\
\hline 1 & 1.15 & $2 \cdot 6$ \\
2 & 1.75 & 625 \\
3 & 2.14 & 925 \\
4 & 2.5 & 12.5 \\
\hline
\end{tabular}

We may use the equation to find at what distance the Board-of-Trade potential-difference is innocuous, and what is the corresponding length of line. Substituting in the fourth equation $V=7, \nu=2$, and $l \nu=6$, I find

$$
y=3 \cdot 28 \text { miles. }
$$

And since $\mathrm{V}=\nu a^{2}$, the corresponding length of line will be $\sqrt{3 \cdot 5}$, or 1.87 miles. Thus in round numbers 2 miles of track carrying a current of 300 amperes with a potentialdifference of 7 volts between the ends might produce a vertical magnetic force of $5 \times 10^{-5}$ units on an observatory at a distance of 3 miles.

If we remember that $\lambda$ is the current required to drive the cars in a mile of line, we can put the rule thus :-

Multiply the current in amperes required to drive the cars 
in a mile of line by the number $\frac{1}{10} \mu^{2} / 8$. Let the result be $\mathrm{J}$ amperes. Then the maximum value of $a$ is given by

$$
2 \cdot 5 \mathrm{~J} \frac{a^{4}}{y} \frac{1}{\sqrt{\left(a^{2}+4 y^{2}\right)}}=1 \text {. }
$$

According to the figures assumed above the value of $\mu^{2} / 8$ is 05 .

Again, we have

\section{Hence}

$$
\begin{aligned}
& h \nu=\lambda \mu^{2}=\frac{\lambda h}{k} . \\
& \lambda=k \nu,
\end{aligned}
$$

as is obvious from the definitions.

The value of $1 / k$ is, as we have seen, $\cdot 0135 \mathrm{ohm}$; and since $\mu^{2}=h / k$, we have

$$
\frac{1}{h}=\frac{1}{\mu^{2} k}=\cdot 034 \mathrm{ohm} \text { approximately. }
$$

Of course if a lower value be assumed for $\mu^{2}$ the value of $1 / h$ is proportionately raised.

Again, for rails of similar cross-section $h$ varies as the linear dimensions of the cross-section, and $k$ as the square of the same. Hence $\mu^{2}$ varies inversely as the linear dimensions, and the leak is reduced for a given current by increasing the area of the rail.

\section{Appendix added January 1901.}

An important paper was read before the German Physical Society in Berlin in June 1899 by J. Edler (Verhandl. des Deutschen physikal. Gesellsch. i. Jahrg. No. 10), describing a series of experiments in which the magnetic disturbances due to a line at Spandau were measured at a number of stations at different distances from the line; and it occurred to me to apply the above theory to the results. The line is approximately straight, and is about 5 kilometres in length. The current is said to have varied between 35 and 150 amperes. I lave assumed in the calculations a mean current of 100 amperes, the voltage being 500 volts. I also assumed the value of the leak-coefficient for a mile of line to be 025 , corresponding to the lesser of the two values for which tables are calculated in the paper. The value of $\mu^{2}$ which corresponds to this leak is, when the distances are measured in kilometres, approximately equal to 08 , and the leak in a length of $a$ kilometres is $01 a^{2}$. The results for the vertical disturbance are given in 'Table III., in which the first column gives the distance of the observing-station from the line in kilometres, the second 
Theory of Magnetic Disturbance by Earth-Currents. 441 column the calculated, and the third the observed values of the force, the unit being as usual $10^{-5}$ Gauss.

TABLE III.

\begin{tabular}{|c|c|c|}
\hline$y$. & F calculated. & F observed. \\
\hline 38 & $12 \cdot 8$ & $23 \cdot 17$ \\
64 & $7 \cdot 3$ & $10 \cdot 6$ \\
.79 & $5 \cdot 8$ & $5 \cdot 6$ \\
.92 & 4.9 & 4.3 \\
3.01 & .99 & .95 \\
7.48 & .21 & .24 \\
\hline 1.54 & 1.5 & 1.04 \\
\hline
\end{tabular}

It is clear that at the two shorter distances the formula fails to represent the facts.

In the case of the next four stations, that is for a range from $\cdot 79$ to $7 \cdot 48$ kilometres, or from half a mile up to five miles, the agreement is practically exact.

In the case of the experiment recorded in the last line the river Havel, which from a small map printed with the paper is apparently nearly half a kilometre in width, and another smaller stream lie between the observing-station and the line. This may possibly account for the discrepancy between theory and experiment.

It is easy from Professor Rücker's theory to calculate the horizontal resultant disturbance. Taking, as in fig. 1, A and $B$ to be the source and sink and $O$ the observatory, we have to find the resultant of two forces $I_{\kappa} / O B$ and $I_{k} / O A$ acting at right angles to $O B$ and $O A$ respectively, in such a way that if the one tends to increase the angle MBO, the other tends to decrease the angle MAO.

This resultant is easily shown to be equal to

$$
\frac{\text { I. } \kappa \cdot a}{r_{1} \cdot r_{2}} \text {. }
$$

To apply this I have assumed the whole leak to take place. at the ends of the line, which is clearly quite an extreme case, and have thus obtained the following table.

Phil. Mag. S. 6. Vol. 1. No. 4. April 1901。 2 G 
TABLE IV.

\begin{tabular}{|c|c|c|}
\hline$y$. & H calculated. & H observed. \\
\hline$\cdot 38$ & $2 \cdot 25$ & 9.5 \\
$\cdot 64$ & $2 \cdot 15$ & 6.09 \\
$\cdot 79$ & 2.08 & 4.67 \\
$\cdot 92$ & 1.92 & 4.31 \\
3.01 & $\cdot 83$ & 1.33 \\
7.48 & $\cdot 21$ & $\cdot 44$ \\
1.54 & 1.56 & 1.44 \\
\hline
\end{tabular}

In this case the discrepancy is very marked. The observed and calculated forces are quantities of the same order ; and that is all that can be said. Possibly this may be due to the very close proximity of the river which runs parallel to the line. The horizontal disturbance is due to the vertical component of the leakage-current. We may possibly look upon the river as an extended sink. Currents which start in a horizontal direction from the side of the rail opposite to the river may be diverted by it, gradually becoming vertical, and finally, after having the direction turned through $180^{\circ}$, passing horizontally, or nearly so, back under the rail and into the river ; or it may be that the water-surface under the ground is not very far below the level of the rails-the observing-stations were in most cases only 3.5 metres above the water-level, - and that in consequence of this the lines of flow are inclined at a less angle to the vertical than they would otherwise be.

The presence of the river ought clearly to affect the horizontal disturbance more than the vertical.

XL. Leakage of Electricity from Charged Bodies at Moderate Temperatures.-II. $\quad B y$ J. C. BeatTie, D.Se., F.R.S.E., Professor of Applied Mathematics and Physics, South African College, Cape Town*

$\$ 7$. TN a previous paper + the rate of leakage of electricity 1 from insulated plates covered with different substances was observed when the plates were charged with positive electricity to a potential of between 200 and 100

* Communicated by the Author. Read before the Royal Society of Edinburgh, July 1900 .

+ Phil. Mag. ser. 5, vol. xlviii. pp. 97-106 (July 1899). 